BMJ Nutrition, Prevention \& Health

\section{Modest effects of dietary supplements during the COVID-19 pandemic: insights from 445850 users of the COVID-19 Symptom Study app}

To cite: Louca P, Murray B Klaser K, et al. Modest effects of dietary supplements during the COVID-19 pandemic: insights from 445 850 users of the COVID-19 Symptom Study app. BMJ Nutrition, Prevention \& Health 2021;4:e000250. doi:10.1136/ bmjnph-2021-000250

- Prepublication history and additional material is published online only. To view, please visit the journal online (http://dx.doi org/10.1136/bmjnph-2021000250).

For numbered affiliations see end of article.

Correspondence to Dr Cristina Menni, Department of Twin Research and Genetic Epidemiology, King's College London, London WC2R 2LS, UK; cristina.menni@kcl.ac.uk

PL, BM, KK, TDS and CM contributed equally.

Received 1 February 2021 Revised 3 March 2021 Accepted 4 March 2021 Published Online First 19 April 2021

Check for updates

(c) Author(s) (or their employer(s)) 2021. Re-use permitted under CC BY. Published by BMJ.

\section{ABSTRACT}

Objectives Dietary supplements may ameliorate SARSCoV-2 infection, although scientific evidence to support such a role is lacking. We investigated whether users of the COVID-19 Symptom Study app who regularly took dietary supplements were less likely to test positive for SARS-CoV-2 infection.

Design App-based community survey.

Setting 445850 subscribers of an app that was launched to enable self-reported information related to SARS-CoV-2 infection for use in the general population in the UK $(n=372720)$, the USA $(n=45757)$ and Sweden $(n=27373)$. Main exposure Self-reported regular dietary supplement usage (constant use during previous 3 months) in the first waves of the pandemic up to 31 July 2020.

Main outcome measures SARS-CoV-2 infection confirmed by viral RNA reverse transcriptase PCR test or serology test before 31 July 2020 .

Results In 372720 UK participants (175652 supplement users and 197068 non-users), those taking probiotics, omega-3 fatty acids, multivitamins or vitamin $\mathrm{D}$ had a lower risk of SARS-CoV-2 infection by $14 \%$ (95\% Cl $(8 \%$ to $19 \%)), 12 \%(95 \% \mathrm{Cl}(8 \%$ to $16 \%)), 13 \%$ (95\% Cl $(10 \%$ to $16 \%))$ and $9 \%(95 \% \mathrm{Cl}(6 \%$ to $12 \%))$, respectively, after adjusting for potential confounders. No effect was observed for those taking vitamin C, zinc or garlic supplements. On stratification by sex, age and body mass index (BMI), the protective associations in individuals taking probiotics, omega- 3 fatty acids, multivitamins and vitamin D were observed in females across all ages and $\mathrm{BMI}$ groups, but were not seen in men. The same overall pattern of association was observed in both the US and Swedish cohorts.

Conclusion In women, we observed a modest but significant association between use of probiotics, omega-3 fatty acid, multivitamin or vitamin D supplements and lower risk of testing positive for SARS-CoV-2. We found no clear benefits for men nor any effect of vitamin C, garlic or zinc. Randomised controlled trials are required to confirm these observational findings before any therapeutic recommendations can be made.

\section{INTRODUCTION}

A number of micronutrients, including vitamins $\mathrm{C}$ and $\mathrm{D}$ and zinc, have been shown to play key roles in supporting immune function $^{12}$ and in reducing risk of respiratory infection. ${ }^{23}$ These nutrients can be obtained from the diet and are available as dietary supplements either alone or as part of multivitamin or multinutrient mixtures. There are many other dietary supplements available including, omega-3 fatty acids ('fish oil'), probiotics and plant isolates like garlic. ${ }^{4}$ The use of specific dietary supplements in both prevention and acute treatment of infection with SARS-CoV-2 has been promoted by prominent medical entertainment personalities on television and social media since the beginning of the current coronavirus pandemic. ${ }^{5}$ The UK supplement market increased by $19.5 \%$ in the period leading up to the national 'lockdown' in early March 2020, ${ }^{6}$ with a $110 \%$ rise in sales of vitamin $\mathrm{C}$ and a $93 \%$ rise in sales of multivitamin supplements. ${ }^{6}$ Likewise, zinc supplement sales increased by $415 \%$ over the 7-day period ending $8 \mathrm{March}$, at the height of COVID-19 concern in the USA. ${ }^{5}$

A biologically plausible role exists for certain vitamins and minerals in immune pathways. ${ }^{1}$ For example, vitamin $\mathrm{D}$ has been suggested to reduce SARS-CoV-2 transmission by enhancing antiviral immunity and to reduce mortality by mitigating the cytokine 
storm linked with severe COVID-19. ${ }^{78}$ Moreover, zinc also supports the function of the immune system ${ }^{9}$ and may have specific antiviral effects. ${ }^{10}$ However, robust evidence to support a role for vitamins and minerals in preventing infection with SARS-CoV-2 is not available. ${ }^{11}$ Any such evidence would need to take into account factors such as socioeconomic status, ethnicity and occupational exposure to the virus as well as the requirement of a large sample size and a clear confirmation of infection.

By using data from the COVID-19 Symptom Study app, ${ }^{12}$ we tested the hypothesis that individuals taking dietary supplements, were at lower risk of testing positive for SARS-CoV-2, during the first wave of the pandemic. We initially examined whether supplement use was associated with SARS-CoV-2 infection among 372720 UK participants who reported having been tested for SARS-CoV-2 using a reverse transcriptase-PCR (RT-PCR) or serologybased test. Next, we used data from 45757 US and 27373 Swedish (SE) app users who also reported tests for SARS-CoV-2 infection to replicate UK findings.

\section{METHODS}

\section{Study setting and participants}

The COVID-19 Symptom Study app was developed by health data company Zoe Global with input from King's College London, the Massachusetts General Hospital, Lund University, Sweden and Uppsala University, Sweden. In the UK, it was launched in English on Tuesday the 24 March 2020; in the USA in English and Spanish on Sunday the 29 March 2020 and in Sweden it was launched in Swedish on 29 April 2020 as previously described. ${ }^{12} 13$ Anyone over 18 years is able to sign up without any restriction. Individuals are also permitted to record information for dependencies under the age of 18 . Here, we included individuals above 16 years of age (online supplemental figure S1). The app enabled self-reported information related to SARS-CoV-2 infection to be captured. On first use, the app recorded self-reported location, age and core health risk factors. With continued use, participants provided daily updates on symptoms, healthcare visits, SARS-CoV-2 test results and if they were self-quarantining or seeking healthcare, including the level of intervention and related outcomes. Individuals without apparent symptoms were also encouraged to use the app. Through direct updates, new or modified questions were added in real-time to capture data to test emerging hypotheses about COVID-19 symptoms and treatments. Here, we analysed data from the 31 July 2020 data dump.

\section{Assessment of exposure}

Starting on the 2 June 2020, in the three countries, via the app, users were retrospectively asked if they had been taking supplements regularly (defined as: $>3$ times a week for at least 3 months) (online supplemental figure S1). Each user was able to fill in the supplements questionnaire only once (see online supplemental table S1 for list of questions). The questionnaire included use of probiotics, garlic, omega-3 fatty acids ('fish oils'), multivitamins, vitamin D, vitamin C or zinc or no intake of any supplement. Supplement use was recorded as yes/no. For all the analyses, the control group consisted of individuals not taking any supplement.

\section{Ascertainment of outcomes}

Participants were asked if they had been tested for COVID-19 using a RT-PCR or serology-based test and the results (none, negative, pending or positive). Our primary outcome was a report of a positive COVID-19 test between the time when they first reported on the COVID-19 Symptom Study app and 31 July 2020. Participants without a positive or negative test result were excluded (online supplemental figure S1).

\section{Ascertainment of covariates}

Covariates including age, sex, body mass index (BMI), smoking, ethnicity, healthcare worker status and presence of comorbidities (ie, cancer, diabetes, eczema, heart disease, lung disease, kidney disease and hay fever) were self-reported via the app. The app also facilitated the index of multiple deprivation (IMD) to be generated from the relevant government websites of the UK, ${ }^{14}$ Scotland ${ }^{15}$ and Wales, ${ }^{16}$ with the most recent IMD available at the time of analysis used. The IMD was then categorised into quintiles within-population, where 1 is the least deprived and 5 is the most deprived. A subset of the UK app users $(\mathrm{n}=234271)$ also completed a second retrospective questionnaire investigating diet quality at two time points. Here, we included data from the "peripandemic' time point described as the previous month (from user access). The questionnaire included the validated Leeds Short Form Food Frequency Questionnaire (FFQ) developed by Cleghorn and collaborators and listed in the Nutritools ( www.nutritools.org) library. ${ }^{17}{ }^{18}$ We computed the validated Diet Quality Index, as previously described. ${ }^{18}$ Briefly, the Diet Quality Index was composed of fruit, vegetable, oily fish, fat and non-milk extrinsic sugar intakes reflecting five dietary components recognised as indicators of a healthy diet. Standard portion sizes were assigned to each food item on the FFQ $^{18}$

\section{Data sharing}

Anonymised research data are shared with third parties via the centre for Health Data Research UK (HDRUK.ac.uk). US investigators are encouraged to coordinate data requests through the COronavirus Pandemic Epidemiology Consortium (www.monganinstitute.org/cope-consortium). Data updates can be found on https://covid.joinzoe.comhttps:// covid.joinzoe.com.

\section{Statistical analysis}

We studied 372720 UK app users (aged 16-90 years) who selfreported information regarding regular dietary supplement usage and outcome of a COVID-19 test. Of these, 23521 individuals tested positive for SARS-CoV-2 and 349199 tested negative. Multivariate logistic regression adjusting for age, sex, BMI and health status at sign-up was applied to investigate the association between supplement use and testing positive for SARS-CoV-2. We then repeated the analyses (i) adjusting for age, sex, BMI, comorbidities (including type 
2 diabetes, cancer, asthma, heart disease, eczema, hay fever, kidney disease and lung disease), IMD, smoking, ethnicity, health worker/carer status and diet quality and (ii) stratifying by sex, age group $(<40,40-60,>60$ years $)$ and BMI categories (normal weight, overweight, obese/morbidly obese).

Replication was conducted in two independent datasets including 45757 US and 27373 SE app users .

All $p$ values presented were two-sided, with statistical significance determined by the Bonferroni-corrected threshold of significance $(p=0.05 / 7=0.007)$. Statistical analysis was performed using Stata V.12 and ExeTera, a Python library developed at KCL to clean and process the raw dataset. ${ }^{19}$

\section{Patient and public involvement}

No patients were directly involved in designing the research question or in conducting the research. No patients were asked for advice on interpretation or writing up the results. There are no plans to involve patients or relevant patient community in dissemination at this moment.

\section{RESULTS}

The demographic characteristics of the study population are presented in table 1 . Briefly, our discovery cohort included 372720 UK app users who reported having had an RT-PCR-based or serology test for SARS-CoV-2 and who completed the app-based dietary supplement questionnaire. The study sample was predominantly female $(66.8 \%)$ and $>50 \%$ were overweight $(\mathrm{BMI} \quad(\mathrm{SD})=26.8$ (5.6) $\mathrm{kg} / \mathrm{m}^{2}$ ).

As shown in table 1, out of the 372720 UK app users, $175652(47 \%)$ self-reported using supplements regularly since the beginning of the pandemic, while 197068 selfreported they were not taking any supplement. This is in line with the UK general population supplement usage as reported in the National Diet and Nutrition Survey. ${ }^{40}$

In the UK cohort, users regularly supplementing their diet (i) with multivitamins had a lower risk of testing positive for SARS-CoV-2 by $13 \%$ (OR $(95 \% \mathrm{CI})=0.87$ (0.84 to $\left.0.90), \mathrm{p}=1.62 \times 10^{-14}\right)$, (ii) with vitamin $\mathrm{D}$ had a lower risk by

Table 1 Demographic characteristics of the study population

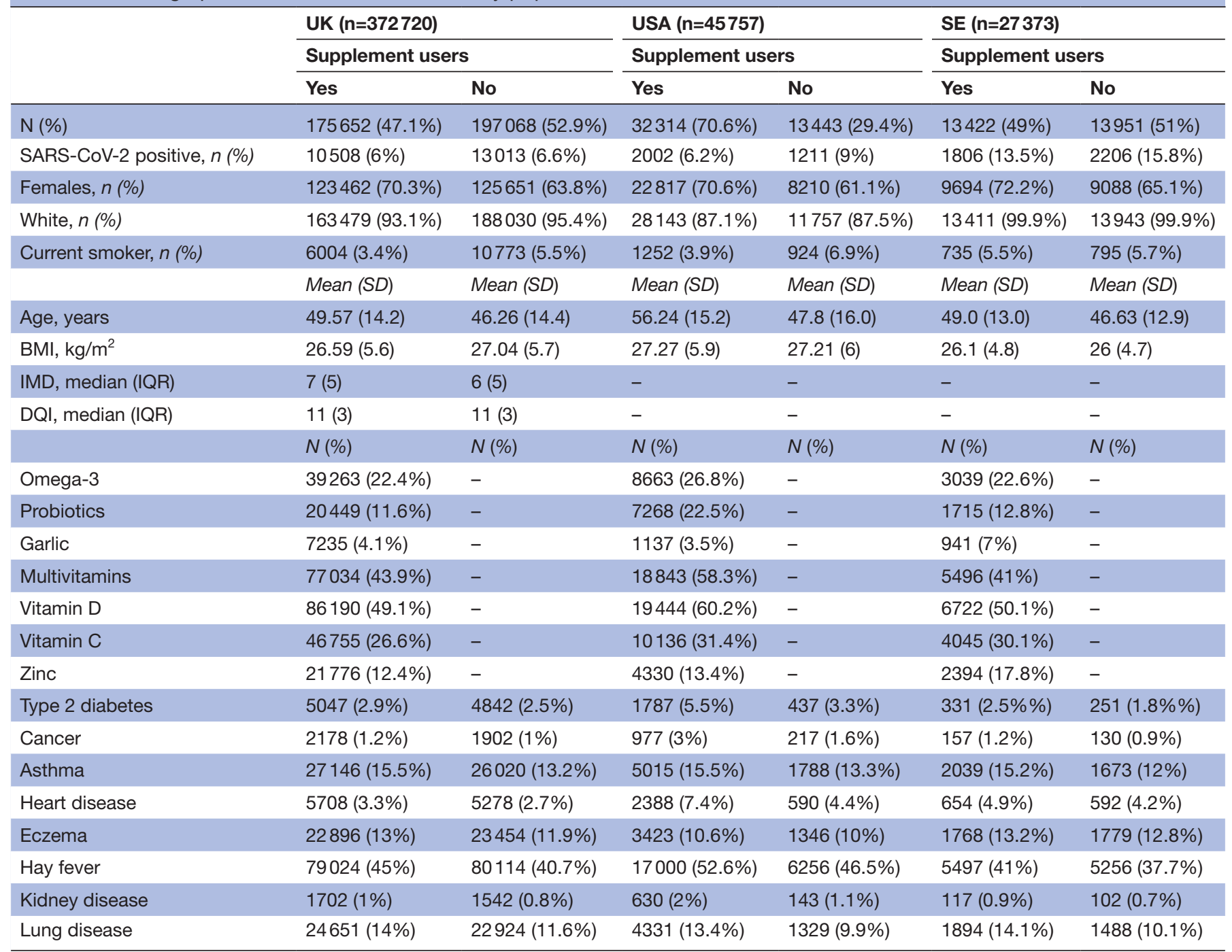

Self-reported comorbidities were not asked at sign-up and therefore not reported by all subjects.

BMI, body mass index; DQI, Diet Quality Score; IMD, index of multiple deprivation; SE, Sweden. 
$9 \%\left(\mathrm{OR}(95 \% \mathrm{CI})=0.91(0.88\right.$ to 0.94$\left.), \mathrm{p}=2.07 \times 10^{-8}\right)$; (iii) with probiotics had a lower risk by $14 \%$ OR $(95 \% \mathrm{CI})=0.86$ (0.81 to 0.92$\left.), p=1.99 \times 10^{-6}\right)$ and (iv) with omega-3 fatty acids had a lower risk by $12 \%(\mathrm{OR}(95 \% \mathrm{CI})=0.88(0.84$ to 0.92$), \mathrm{p}=5.8 \times 10^{-8}$ ), after adjusting for age, sex, BMI, sign-up health status and multiple testing (figure 1). There were no significant associations in those supplementing with zinc, vitamin $\mathrm{C}$ or garlic (figure 1). Crude results are presented in online supplemental table S2. To account for a potential healthy user bias, we did a sensitivity analysis further adjusting for ethnicity, comorbidities, smoking, IMD and health worker/carer status, results remained consistent with the previous analysis (figure 1), although the effect of probiotics appeared weaker. We also adjusted for the Diet Quality Index. In line with the literature, ${ }^{21}$ we found diet quality and supplements usage to be positively correlated $(\mathrm{r}=0.07, \mathrm{p}<0.0001)$, supporting that people whose diet are closer to the recommended one are more likely to take supplements compared with those whose diet is far from the recommendation. However, when we adjust for diet quality, results remain consistent, suggesting that the effect of supplement use is independent from the effect of diet quality (figure 1).

Next, we ran the analyses stratifying by sex, age group and BMI categories and we detected sexual dimorphism in the association between supplement use and testing positive for SARS-CoV-2 (figure 1). Females taking probiotics, omega-3 fatty acids, multivitamins and vitamin D had a lower risk of infection across all age groups and
BMI categories $(\mathrm{OR}(95 \% \mathrm{CI})$ ranging from 0.73 (0.63 to 0.85 ) for probiotics in women $<40$ years of age to 0.91 (0.86 to 0.96 ) for vitamin $\mathrm{D}$ in women aged between 40 and 60 years). No protective association was observed in males overall. However, in post hoc subgroup analyses, men aged $<40$ years or normal weight and taking multivitamins and men aged 40-60 years and taking omega-3 fatty acids were less likely to be infected (figure 1). In contrast, there was a positive association in men aged $>60$ years taking zinc $\left(1.4\left(1.16\right.\right.$ to1.69), $\left.\mathrm{p}=4.92 \times 10^{-4}\right)$ or vitamin $\mathrm{C}$ supplements (1.22 (1.05 to 1.41$), \mathrm{p}=8.1 \times 10^{-3}$ ) (figure 1) for testing positive for SARS-CoV-2.

To replicate significant findings from the UK cohort, we next used data from the 45757 US and $27373 \mathrm{SE}$ app users. Cohorts were similar, in that they were also predominantly female (USA: $67.8 \%$, SE: $68.6 \%$ ) and a greater proportion were overweight (BMI (SD), USA: $27.3(5.9) \mathrm{kg} / \mathrm{m}^{2}$, SE: $\left.26(4.7) \mathrm{kg} / \mathrm{m}^{2}\right)$. Overall UK findings were mirrored in both cohorts (figure 2). However, findings by gender varied different in different cohorts (figure 2).

Associations in females were replicated in the US cohort, but omega-3 supplement use was not associated with testing positive in Swedish females (figure 2). US males using probiotics or vitamin $\mathrm{D}$ had a decreased risk of a positive test for SARS-CoV-2 (figure 2), while Swedish men taking probiotics, omega-3 fatty acids, multivitamins or vitamin D had a decreased risk of infection (figure 2).

\section{Supplements and COVID-19}

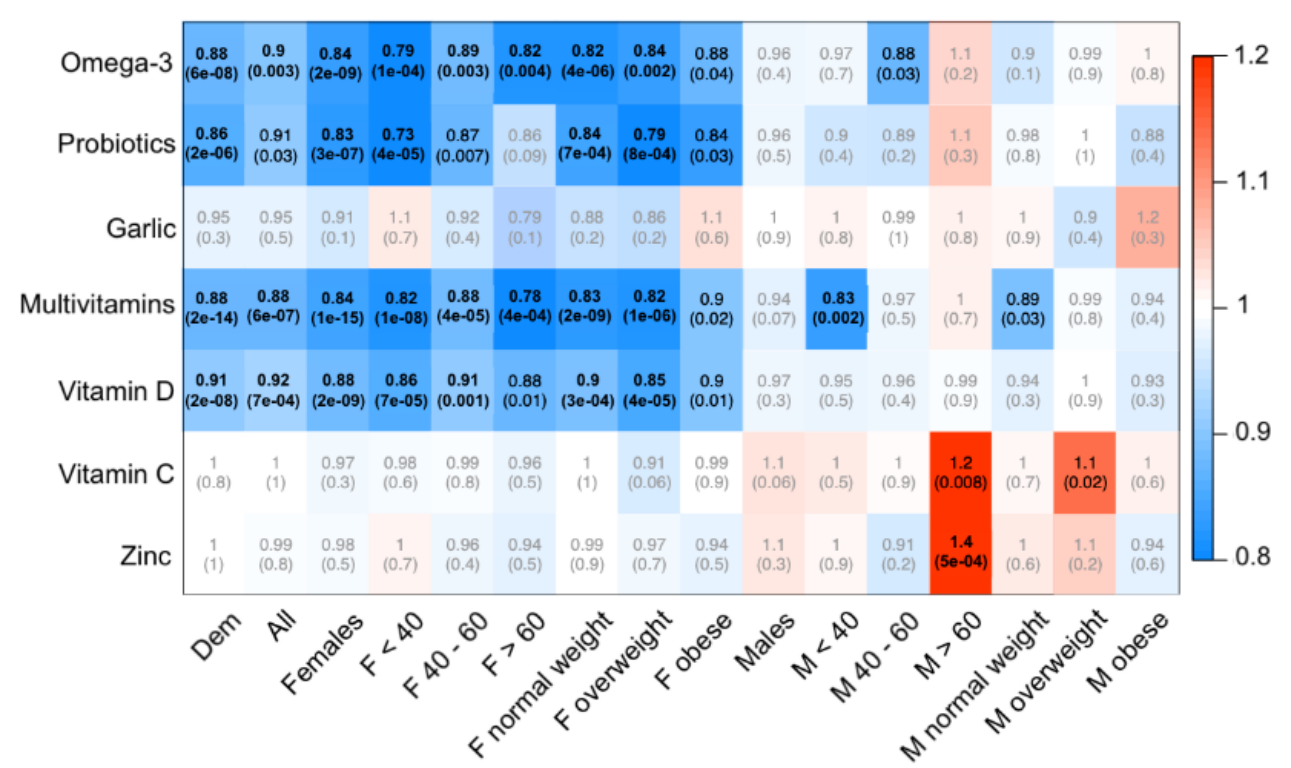

Figure 1 Associations between testing positive for SARS-CoV-2 and self-reported use of supplements in UK app users. Each cell of the matrix displays the OR of the association between use of a type of supplement and testing positive with the corresponding $\mathrm{p}$ value in parentheses. The table is colour coded according to the OR, with blue denoting a reduced risk and red denoting an increased risk of testing positive. Bold entries are statistically significant after accounting for multiple testing using Bonferroni correction. Dem, adjusted for age, sex, body mass ndex (BMI) and health status at sign up; All, adjusted for Dem, index of multiple deprivation, ethnicity, comorbidities (type 2 diabetes, cancer, asthma, heart disease, eczema, hay fever, kidney disease and lung disease), smoking, diet quality; stratified analyses are adjusted for age, (BMI) and health status at sign up as appropriate. 


\section{DISCUSSION}

In the largest observational study on SARS-CoV-2 infection and dietary supplement use to date on over 400000 app users from three different countries, we show a significant association between users of omega-3 fatty acid, probiotic, multivitamin or vitamin D supplements and lower risk of testing positive for infection with SARS-CoV-2. However, our stratified analysis in the tested group shows a strong sexual dimorphism with the consistent protective effect present only in females, at least in the UK, and for some supplements in the USA and SE. This association has several potential explanations including: (i) biological explanations include discordant immune systems between sexes that could respond differently to supplements. ${ }^{22}$ Indeed, a sexual dimorphism in nutrient metabolism has been previously reported, with females having a more robust immune response than men. ${ }^{23}$ Moreover, females typically possess a more resilient immune system than males with higher numbers of circulating B cells when matched for age, BMI and clinical parameters, ${ }^{24}$ as well as a slower age-related decline in circulating $\mathrm{T}$ cells and B cells. ${ }^{24}$ It is therefore plausible that supplements could better support the immune system of females than males, although the lack of consistency between countries is problematic; (ii) differences in body weight and body composition between males and females meaning that supplement dosing on a per body weight basis may be higher in females ${ }^{25}$; (iii) residual confounding due to sex differences in health-related behaviours, including COVID-19. ${ }^{26}$ Polling reveals that a greater percentage of females versus males are anxious for the health of themselves or their family and therefore are more precautionary, cancelling plans and staying home more often. ${ }^{26}$ Females who purchase vitamins may also be more health conscious than males, such as having greater use of wearing face masks and hand-washing. ${ }^{27-29}$ Indeed, in our data, we found that women tended to wear masks more often than males $(44 \%$ of women report wearing a mask at least some of the time when outside, compared with $36 \%$ of men, $\mathrm{p}<0.001)$.

\section{Vitamin D}

A potential antimicrobial role of vitamin $\mathrm{D}$ in infections dates back almost a century, ${ }^{30}$ with several mechanistic study supporting a positive physiological role. ${ }^{3}$ Immune cells express the vitamin D receptor and some can synthesise the active form of vitamin D. Vitamin D influences the function of antigen-presenting cells, $T$ cells and $\mathrm{B}$ cells. $^{31}$ It also promotes production of cathelicidin, a microbicidal component of the innate immune system. ${ }^{32}$ The overlap between risk factors for vitamin D deficiency and risk of severe COVID-19, such as obesity, age and ethnicity, gives some plausibility to a protective role of vitamin D. ${ }^{30}$ A meta-analysis of 39 randomised controlled trials reported that vitamin $\mathrm{D}$ reduces the risk of respiratory infections by around $11 \%$, but there was considerable heterogeneity. ${ }^{33}$ However, a Mendelian randomisation study suggests that genetic levels of vitamin D are not associated with COVID-19 susceptibility, ${ }^{34}$ in line with recent results from the UKBiobank. ${ }^{35}$

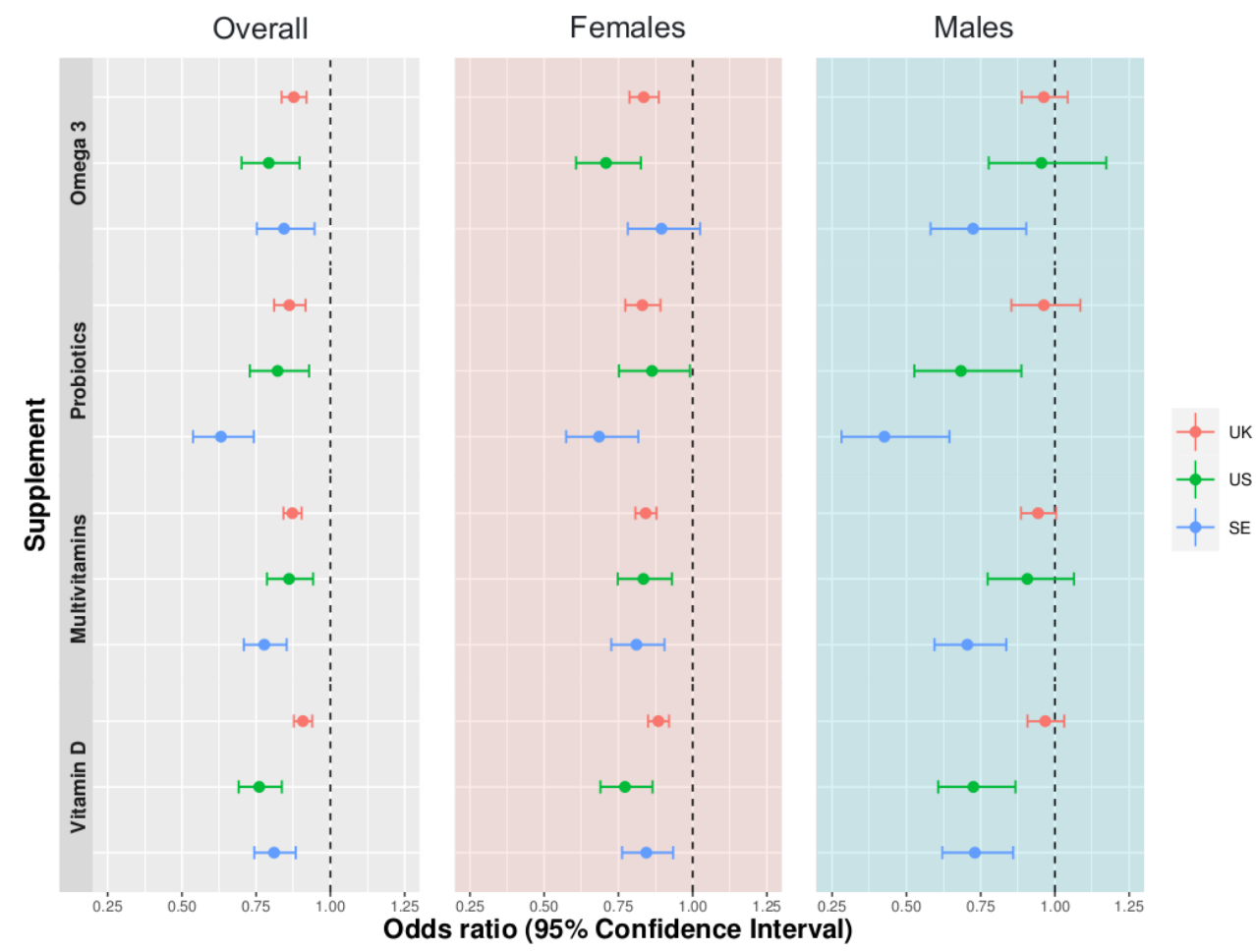

Figure 2 ORs and 95\% Cls for the associations between testing positive for SARS-CoV-2 and self-reported use of supplements in three cohorts $(n=372720$ UK, $n=45575$ USA and $n=27373$ SE). Overall sample analyses are adjusted for age, sex, body mass index (BMI) and health status at sign up. Analyses according to sex are adjusted for age, BMI and health status at sign up. 
In our data, we find a modest protective effect for infection, with a $9 \%$ reduction in risk of testing positive for SARS-CoV-2 in the overall UK cohort, $24 \%$ in the US cohort and $19 \%$ in the SE group.

\section{Multivitamins}

Multivitamin supplements typically include multiple vitamins and multiple minerals including trace elements ${ }^{36}$; many of these have antioxidant properties and roles in supporting the immune system. ${ }^{12}$ Specific micronutrient deficiencies, including zinc, selenium, vitamin A, vitamin $\mathrm{D}$ and vitamin $\mathrm{E}$, have been shown to be detrimental during viral infections. ${ }^{37-39}$ Although some randomised controlled trials have shown that multivitamin supplements reduce the risk of respiratory infections, ${ }^{40}$ a recent review argues that this evidence is weak and unclear. ${ }^{41}$ Here, we provide evidence to support a modest protective effect in those taking multivitamin supplements similar to vitamin $\mathrm{D}$ with a $13 \%$ reduction in risk of testing positive for SARS-CoV-2 in the overall UK cohort, $12 \%$ in the US cohort and $22 \%$ in the SE cohort.

\section{0mega-3 fatty acids}

Omega-3 fatty acids can influence antigen-presenting cell, T-cell and B-cell function, although their effects on these cell types in humans is not consistently reported. However, they are clearly demonstrated to be anti-inflammatory ${ }^{42}$ and to be converted to specialised proresolving mediators such as resolvins, protectins and maresins. ${ }^{43}$ Whether this is a mechanism by which they reduce risk of testing positive for SARS-CoV-2 is not clear. Nevertheless, here we provide evidence to support a protective effect in omega-3 fatty acid supplements users with a $12 \%$ reduction in risk of testing positive for SARS-CoV-2 in the overall UK cohort, $21 \%$ in the US cohort and $16 \%$ in the SE cohort. Although, in the UK, stratified analysis shows this effect is largely driven by females, and only significant in one male stratum.

\section{Probiotics}

Probiotics modify the host's gut microbiota and may generate antiviral metabolites, and they interact with the host's gut-associated immune system. ${ }^{44}$ This can result in improved immunity, including enhanced responses to the seasonal influenza vaccine. ${ }^{45}$ Mechanistic studies support a gut-lung axis, ${ }^{46}$ whereby immune effects of microbiota at the gut level can be transferred to the lung, most likely through movement of immune cells. This could explain why some probiotic organisms reduce risk $^{47-49}$ and severity ${ }^{50}$ of respiratory tract infections. Here, we provide evidence to suggest that people taking probiotics supplements are modestly protected with a $14 \%$ reduction in risk of testing positive for SARS-CoV-2 in the overall UK cohort, $18 \%$ in the US cohort and $37 \%$ in the SE cohort. However, effects of probiotics are strain and species specific and we have no information of which probiotics or their quality were being used by participants in this study. Moreover, when we adjusted for other covariates including diet, the effect of probiotics was weaker suggesting that probiotic use may be confounded by a healthy diet.

\section{Zinc, vitamin $\mathrm{C}$ and garlic}

We saw no protective effects of zinc, garlic or vitamin C. Both zinc and vitamin $\mathrm{C}$ have been previously suggested to support the immune system and to prevent respiratory infections. ${ }^{1251}$ Although, their efficacy and evidence base has been questioned and a meta-analysis of vitamin $\mathrm{C}$ showed no preventive benefit but a reduction in severity and a modest reduction in symptom duration. ${ }^{52} 53$

Strengths of our study include its large sample size, the confirmation of SARS-CoV-2 through a RT-PCR-based or serology-based test, and the replication of the key findings from the UK cohort in two other cohorts, one in the US cohort and the other in SE cohort. Furthermore, we had information on diet quality. Indeed, consistent with the literature, ${ }^{21}$ in our data we found a positive correlation between diet quality and supplements usage suggesting that people with a healthier diet were more likely to take supplements. However, we were able to adjust for diet quality and showed that the effect of supplements is independent from the effect of diet quality.

Our study also has a number of limitations. First, we used self-reported data which can introduce information bias, including misclassification, or effect bias exposure if participants started taking supplements after developing symptoms. There is also a possibility of participants having COVID-19 symptoms (and likely having COVID-19) but not having had any test because of low testing capacity ${ }^{54}$ and then started taking supplements. We believe this is possible, although would have reduced any real effect. To help mitigate potential spurious associations, we adjusted for self-reported health status at sign-up. Second, participants using the app were a self-selected group and may not be fully representative of the general population. Indeed, our study population is predominantly female, although supplement usage and BMI are in line with data for the UK general population. ${ }^{40}$ This may cause collider bias, that is, both use of supplements and having COVID-19 might influence the probability of an individual participating in the COVID-19 Symptom Study, and this selection effect could introduce an apparent association between supplement use and COVID-19 status. ${ }^{55}$ Third, the SARS-CoV-2 infection diagnosis was mainly based on the RT-PCR test that has $<100 \%$ sensitivity (true positive rate) ${ }^{5657}$ Fourth, participants might have been taking supplements in addition to the seven we asked them about, and we were not able to account for multiple sources of vitamins/minerals. Fifth, malnutrition is a significant risk factor for a severe form of COVID-19 infection, ${ }^{58}$ however we had information on diet, we were unable to infer vitamin/mineral deficiencies. Sixth, we do not know the exact intakes of the ingredients within the supplements used by the participants, nor we had any information regarding dosage and hence we were unable to link supplements dosage or intakes from multiple sources with disease outcome. 
Furthermore, within the useful timeframe, the app has not captured information on behaviours such as washing hands, use of disinfectants or social distancing and only a proportion of participants replied to the questions on use of masks. We also were only able to adjust for a crude, area-based, measure of deprivation rather than individually based measures of socioeconomic background, such as educational attainment, occupation and income; thus, residual confounding by socioeconomic status is possible. Finally, this is observational data captured during a specific timeframe, and our study design does not allow an inference of causality.

In conclusion, our data show that women taking multivitamins, omega- 3 fatty acids, vitamin $\mathrm{D}$ or probiotics have a slightly lower risk of SARS-CoV-2 infection in the UK, US and SE cohorts, but no effect in those taking zinc, vitamin $\mathrm{C}$ or garlic. Given the interest in supplements during the pandemic, large randomised controlled trials of selected supplements testing their protective effects, and also possible adverse effects, on disease severity are required before any evidence-based recommendations can be made. We eagerly await the result of ongoing trials, including of vitamin $\mathrm{D}$, omega-3 fatty acids and probiotics and COVID-19 risk. ${ }^{59}$

\section{What this paper adds}

\section{What is already known on this topic}

- Dietary supplements have been shown to play key roles in supporting immune function, but the extent to which specific supplements are associated with reduced risk of SARS-CoV-2 infection is not known.

\section{What this study adds}

- Individuals taking multivitamins, omega-3 fatty acids, probiotics or vitamin D were less likely to be tested positive for SARS-CoV-2 in three large independent cohorts of app users.

- There was a significant protective association for vitamin D, omega-3 fatty acids, probiotics and multivitamins in female users across all ages and body mass index categories within the largest (UK) cohort; yet, there was no association in male users of this cohort.

- Vitamin C, zinc and garlic supplements had no association with risk for SARS-CoV-2.

- There is a need for randomised controlled trials of selected supplements.

\section{Author affiliations}

${ }^{1}$ Department of Twin Research and Genetic Epidemiology, King's College London, London, UK

${ }^{2}$ School of Biomedical Engineering \& Imaging Sciences, King's College London, London, UK

${ }^{3}$ Clinical \& Translational Epidemiology Unit, Massachusetts General Hospital, Boston, Massachusetts, USA

${ }^{4}$ Department of Clinical Sciences, Lund University, Lund, Sweden

${ }^{5}$ MRC Unit for Lifelong Health and Ageing at UCL, University College London,

London, UK

${ }^{6}$ Department of Nutritional Sciences, King's College London, London, UK

${ }^{7}$ Zoe Global Limited, London, UK

${ }^{8}$ Division of Rheumatology, Orthopaedics and Dermatology, School of Medicine, University of Nottingham, Nottingham, UK

${ }^{9}$ Human Development \& Health, Faculty of Medicine, University of Southampton, Southampton, UK
Correction notice The article has been corrected since it was published online first. This is to inform that PL, BM, KK, TDS and CM shares equal contributorship.

Twitter Ellen Thompson @EllenJoT

Acknowledgements Zoe provided in kind support for all aspects of building, running and supporting the app and service to all users worldwide. The Department of Twin Research is funded by the Wellcome Trust, Medical Research Council, European Union, Chronic Disease Research Foundation (CDRF), Zoe Global Ltd and the National Institute for Health Research (NIHR)-funded BioResource, Clinical Research Facility and Biomedical Research Centre based at Guy's and St Thomas' NHS Foundation Trust in partnership with King's College London. CM is funded by the Chronic Disease Research Foundation and by the MRC Aim-Hy project grant. $\mathrm{PL}$ is funded by the CDRF, $\mathrm{S} 0$ is funded by the Wellcome/EPSRC Centre for Medical Engineering (WT203148/Z/16/Z), Wellcome Flagship Programme (WT213038/ Z/18/Z), and PCC is supported by the National Institute for Health Research Southampton Biomedical Research Centre. We express our sincere thanks to all the participants of the COVID Symptom Study app including study volunteers enrolled in cohorts within the Coronavirus Pandemic Epidemiology (COPE) consortium. We thank the staff of Zoe Global Limited, the Department of Twin Research at King's College London, the Clinical \& Translational Epidemiology Unit at Massachusetts General Hospital, Researchers and staff at Lund University in Sweden for their tireless work in contributing to the running of the study and data collection.

Contributors Funding acquisition: JW, TDS. Conceptualisation: CM, TDS. Formal analysis: BM, PL, KK, CM. Data curation: BM, MG. Resources: MSG, RB, MM, ERL, OM, ET, RC, CJS, SO, SEB, DAD, LHN, JM, MG, PWF, ATC, RG, CHS. Wrote original draft: PL, AMV, PCC, CM. Revised the manuscript: all. CM and TDS are responsible for the overall content as guarantors. The guarantors accept full responsibility for the work and/or the conduct of the study, had access to the data and controlled the decision to publish. The manuscript's guarantors affirm that the manuscript is an honest, accurate and transparent account of the study being reported; that no important aspects of the study have been omitted and that any discrepancies from the study as planned have been explained. The corresponding author attests that all listed authors meet authorship criteria and that no others meeting the criteria have been omitted.

Funding This work was supported by Zoe Global Limited. The Department of Twin Research receives grant support from the Wellcome Trust (212904/Z/18/Z) and the Medical Research Council (MRC)/British Heart Foundation Ancestry and Biological Informative Markers for Stratification of Hypertension (AIMHY; MR/M016560/1), European Union, Chronic Disease Research Foundation (CDRF), Zoe Global Ltd, NIH and the National Institute for Health Research (NIHR)-funded BioResource, Clinical Research Facility and Biomedical Research Centre based at Guy's and St Thomas' NHS Foundation Trust in partnership with King's College London. PL is funded by the Chronic Disease Research Foundation; AMV is supported by the National Institute for Health Research Nottingham Biomedical Research Centre. CHS is an Alzheimer's Society Junior Fellowship AS-JF-17-011; S0 is funded by the Wellcome/EPSRC Centre for Medical Engineering (WT203148/Z/16/Z), Wellcome Flagship Programme (WT213038/Z/18/Z). ATC is the Stuart and Suzanne Steele MGH Research Scholar and is a Team Leader for the Stand Up to Cancer Foundation. ATC, LHN, JAM and DAD are supported by the Massachusetts Consortium on Pathogen Readiness (MassCPR). CM is funded by the Chronic Disease Research Foundation and by the MRC Aim-Hy project grant. PCC is supported by the National Institute for Health Research Southampton Biomedical Research Centre. MSG is supported by the Wellcome Flagship Programme (WT213038/Z/18/Z). MFG and PWF receive support from the Swedish Research Council, Swedish Heart-Lung Foundation and the Swedish Foundation for Strategic Research (LUDC-IRC 15-0067).

Competing interests TDS, AMV, ERL and SEB are consultants to Zoe Global Limited ('Zoe'). JW is an employee of Zoe. PCC has research funding from BASF AS and Bayer Consumer Care; is an advisor/consultant to BASF AS, DSM, Danone/ Nutricia, Cargill, Smartfish, Nutrileads, Bayer Consumer Care and Pfizer (now GSK) Consumer Healthcare and has received travel reimbursement/speaking fees from Danone, Fresenius Kabi, Pfizer (now GSK) Consumer Healthcare, Smartfish, Biogredia and the California Walnut Commission. ATC has received consulting fees from Bayer Pharma, Pfizer and Boehringer Ingelheim.

Patient consent for publication Not required.

Ethics approval Ethical approval for use of the app for research purposes in the UK was obtained from King's College London Ethics Committee (review reference LRS-19/20-18210) and all users provided consent for non-commercial use. The US protocol was approved by the Partners Human Research Committee (protocol 2020P000909). The Swedish protocol was approved by the Swedish Ethical Review Authority (protocol 2020-01803). 
Provenance and peer review Not commissioned; externally peer reviewed by Emily Sonestedt, Lund University, Sweden.

Data availability statement Data are available on reasonable request. Anonymised research data are shared with third parties via the centre for Health Data Research UK (HDRUK.ac.uk). US investigators are encouraged to coordinate data requests through the COPE Consortium (www.monganinstitute.org/copeconsortium). Data updates can be found on https://covid.joinzoe.com.

Supplemental material This content has been supplied by the author(s). It has not been vetted by BMJ Publishing Group Limited (BMJ) and may not have been peer-reviewed. Any opinions or recommendations discussed are solely those of the author(s) and are not endorsed by BMJ. BMJ disclaims all liability and responsibility arising from any reliance placed on the content. Where the content includes any translated material, BMJ does not warrant the accuracy and reliability of the translations (including but not limited to local regulations, clinical guidelines, terminology, drug names and drug dosages), and is not responsible for any error and/or omissions arising from translation and adaptation or otherwise.

Open access This is an open access article distributed in accordance with the Creative Commons Attribution 4.0 Unported (CC BY 4.0) license, which permits others to copy, redistribute, remix, transform and build upon this work for any purpose, provided the original work is properly cited, a link to the licence is given, and indication of whether changes were made. See: https://creativecommons.org/ licenses/by/4.0/.

\section{ORCID iDs}

Panayiotis Louca http://orcid.org/0000-0001-5956-1433 Benjamin Murray http://orcid.org/0000-0002-8198-8131 Kerstin Klaser http://orcid.org/0000-0002-5230-6939

Mark S Graham http://orcid.org/0000-0002-4170-1095 Mohsen Mazidi http://orcid.org/0000-0003-3059-229X Emily R Leeming http://orcid.org/0000-0002-0531-4901 Ellen Thompson http://orcid.org/0000-0003-2118-821X Ruth Bowyer http://orcid.org/0000-0002-6941-8160 David A Drew http://orcid.org/0000-0002-8813-0816 Long H Nguyen http://orcid.org/0000-0002-5436-4219 Jordi Merino http://orcid.org/0000-0001-8312-1438 Maria Gomez http://orcid.org/0000-0001-6210-3142 Olatz Mompeo http://orcid.org/0000-0002-1754-6136 Ricardo Costeira http://orcid.org/0000-0002-8316-5219 Carole H Sudre http://orcid.org/0000-0001-5753-428X Rachel Gibson http://orcid.org/0000-0002-5823-6468 Claire J Steves http://orcid.org/0000-0002-4910-0489 Jonathan Wolf http://orcid.org/0000-0002-0530-2257 Paul W Franks http://orcid.org/0000-0002-0520-7604 Sebastien Ourselin http://orcid.org/0000-0002-5694-5340 Andrew T Chan http://orcid.org/0000-0001-7284-6767 Sarah E Berry http://orcid.org/0000-0002-5819-5109 Ana M Valdes http://orcid.org/0000-0003-1141-4471 Philip C Calder http://orcid.org/0000-0002-6038-710X Tim D Spector http://orcid.org/0000-0002-9795-0365 Cristina Menni http://orcid.org/0000-0001-9790-0571

\section{REFERENCES}

1 Gombart AF, Pierre A, Maggini S. A review of micronutrients and the immune System-Working in harmony to reduce the risk of infection. Nutrients 2020;12:236.

2 Calder PC. Nutrition, immunity and COVID-19. BMJ Nutr Prev Health 2020;3:e000085:74-92.

3 Martineau AR, Jolliffe DA, Hooper RL, et al. Vitamin D supplementation to prevent acute respiratory tract infections: systematic review and meta-analysis of individual participant data. BMJ 2017;356:i6583.

4 Lentjes MAH. The balance between food and dietary supplements in the general population. Proc Nutr Soc 2019;78:97-109.

5 Adams KK, Baker WL, Sobieraj DM. Myth Busters: dietary supplements and COVID-19. Ann Pharmacother 2020;54:820-6.

6 Kantar. Consumer panel for food, beverages and household products, 2020. Available: kantarworldpanel.com [Accessed 6 Oct 2020].

7 Grant WB, Lahore H, McDonnell SL, et al. Evidence that vitamin D supplementation could reduce risk of influenza and COVID-19 infections and deaths. Nutrients 2020;12:988.
8 Khare D, Godbole NM, Pawar SD, et al. Calcitriol [1, 25[OH]2 D3] pre- and post-treatment suppresses inflammatory response to influenza A (H1N1) infection in human lung A549 epithelial cells. Eur J Nutr 2013;52:1405-15.

9 Wessels I, Maywald M, Rink L. Zinc as a gatekeeper of immune function. Nutrients 2017;9. doi:10.3390/nu9121286. [Epub ahead of print: 25 Nov 2017].

10 Read SA, Obeid S, Ahlenstiel C, et al. The role of zinc in antiviral immunity. Adv Nutr 2019;10:696-710.

11 Cheng VC-C, Wong S-C, Yuen K-Y, et al. Infection risk in health care workers. JAMA Netw Open 2019;2020:e209687

12 Menni C, Valdes AM, Freidin MB, et al. Real-Time tracking of self-reported symptoms to predict potential COVID-19. Nat Med 2020;26:1037-40.

13 Drew DA, Nguyen LH, Steves CJ, et al. Rapid implementation of mobile technology for real-time epidemiology of COVID-19. Science 2020;368:1362-7.

14 GOV.UK. English indices of deprivation 2019. GOV.UK, 2019. Available: https://www.gov.uk/government/statistics/english-indicesof-deprivation-2019 [Accessed 25 Nov 2020].

15 Scottish Government SAH. Scottish index of multiple deprivation, 2016. Available: http://www2.gov.scot/Topics/Statistics/SIMD [Accessed 25 Nov 2020].

16 StatsWales. WIMD 2019, 2019. Available: https://statswales.gov. wales/Catalogue/Community-Safety-and-Social-Inclusion/WelshIndex-of-Multiple-Deprivation/WIMD-2019 [Accessed 25 Nov 2020].

17 Nutritools. Tool information: short form FFQ, 2017. Available: https:// www.nutritools.org/tools/136 [Accessed 15 Oct 2020].

18 Mazidii M, Leeming E, Merino J. Impact of COVID-19 on health behaviours and body weight: a prospective observational study in a cohort of 1.1 million UK and US individuals. Nature Portfolio (Published Online First: 26 February 2021).

19 Murray B. exetera 0.2.8.dev1: High-volume key-value store and analytics, based on hdf5, 2020. Available: https://github.com/kclbmeis/zoe-data-store [Accessed 19 Oct 2020].

20 Henderson L, Swan J, Gregory J. The National Diet \& Nutrition Survey : adults aged 19 to 64 years. Vitamin and mineral intake and urinary analytes 2003.

21 Anders S, Schroeter C. The impact of nutritional supplement intake on diet behavior and obesity outcomes. PLoS One 2017:12:e0185258.

22 Giefing-Kröll C, Berger P, Lepperdinger G, et al. How sex and age affect immune responses, susceptibility to infections, and response to vaccination. Aging Cell 2015;14:309-21.

23 Raposo SE, Fondell E, Ström P, et al. Intake of vitamin C, vitamin $\mathrm{E}$, selenium, zinc and polyunsaturated fatty acids and upper respiratory tract infection-a prospective cohort study. Eur J Clin Nutr 2017;71:450-7

24 Márquez EJ, Chung C-H, Marches R, et al. Sexual-dimorphism in human immune system aging. Nat Commun 2020;11:751.

25 Rogers AR, Mukherjee A. Quantitative genetics of sexual dimorphism in human body size. Evolution 1992;46:226-34.

26 Frederiksen B, Gomez I, Salganicoff A. Coronavirus: a look at gender differences in awareness and actions. Kaiser Family FoundationWomen's Health Policy 2020.

27 Guzek D, Skolmowska D, Głąbska D. Analysis of gender-dependent personal protective behaviors in a national sample: polish adolescents' COVID-19 experience (PLACE-19) study. Int J Environ Res Public Health 2020;17:5770.

28 van de Mortel T, Bourke R, McLoughlin J, et al. Gender influences handwashing rates in the critical care unit. Am J Infect Control 2001;29:395-9.

29 Zhong B-L, Luo W, Li H-M, et al. Knowledge, attitudes, and practices towards COVID-19 among Chinese residents during the rapid rise period of the COVID-19 outbreak: a quick online cross-sectional survey. Int J Biol Sci 2020;16:1745-52.

30 Martineau AR, Forouhi NG. Vitamin D for COVID-19: a case to answer? Lancet Diabetes Endocrinol 2020;8:735-6.

31 Prietl B, Treiber G, Pieber TR, et al. Vitamin D and immune function. Nutrients 2013:5:2502-21.

32 Yamshchikov AV, Desai NS, Blumberg HM, et al. Vitamin D for treatment and prevention of infectious diseases: a systematic review of randomized controlled trials. Endocr Pract 2009;15:438-49.

33 Jolliffe DA, Camargo CA, Sluyter JD, et al. Vitamin D supplementation to prevent acute respiratory infections: systematic review and meta-analysis of aggregate data from randomised controlled trials. medRxiv 2020. doi:10.1101/2020.07.14.20152728. [Epub ahead of print: 25 Nov 2020].

34 Butler-Laporte G, Nakanishi T, Mooser V. Vitamin D and Covid-19 susceptibility and severity: a Mendelian randomization study. medRxiv 2020. 
35 Hastie CE, Pell JP, Sattar N. Vitamin D and COVID-19 infection and mortality in UK Biobank. Eur J Nutr.

36 Mulholland CA, Benford DJ. What is known about the safety of multivitamin-multimineral supplements for the generally healthy population? theoretical basis for harm. Am J Clin Nutr 2007;85:318S-22.

37 Caccialanza R, Laviano A, Lobascio F, et al. Early nutritional supplementation in non-critically ill patients hospitalized for the 2019 novel coronavirus disease (COVID-19): rationale and feasibility of a shared pragmatic protocol. Nutrition 2020;74:110835

38 Lee H, Ko G. Antiviral effect of vitamin A on norovirus infection via modulation of the gut microbiome. Sci Rep 2016;6:25835.

39 Weger-Lucarelli J, Carrau L, Levi LI. Host nutritional status affects alphavirus virulence, transmission, and evolution. PLoS Pathog 2019;15:e1008989.

40 Winkler P, de Vrese M, Laue C, et al. Effect of a dietary supplement containing probiotic bacteria plus vitamins and minerals on common cold infections and cellular immune parameters. Int J Clin Pharmacol Ther 2005;43:318-26.

41 Cramer H, Hannan N, Schloss J. Multivitamins for acute respiratory tract infections: a rapid review. Adv Integr Med.

42 Calder PC. $n$-3 PUFA and inflammation: from membrane to nucleus and from bench to bedside. Proc Nutr Soc 2020:1-13.

43 Calder PC. Eicosapentaenoic and docosahexaenoic acid derived specialised pro-resolving mediators: concentrations in humans and the effects of age, sex, disease and increased omega-3 fatty acid intake. Biochimie 2020;178:105-23.

44 Ahern PP, Maloy KJ. Understanding immune-microbiota interactions in the intestine. Immunology 2020;159:4-14.

45 Yeh T-L, Shih P-C, Liu S-J, et al. The influence of prebiotic or probiotic supplementation on antibody titers after influenza vaccination: a systematic review and meta-analysis of randomized controlled trials. Drug Des Devel Ther 2018;12:217-30.

46 Clarke TB. Early innate immunity to bacterial infection in the lung is regulated systemically by the commensal microbiota via NOD-like receptor ligands. Infect Immun 2014;82:4596-606.

47 Vouloumanou EK, Makris GC, Karageorgopoulos DE, et al. Probiotics for the prevention of respiratory tract infections: a systematic review. Int J Antimicrob Agents 2009;34:197.e1-197.e10.
48 Liu K-xiong, Zhu Y-gang, Zhang J, Liu K, Zhu Y, et al. Probiotics' effects on the incidence of nosocomial pneumonia in critically ill patients: a systematic review and meta-analysis. Crit Care 2012;16:R109.

49 Hao Q, Lu Z, Dong BR, et al. Probiotics for preventing acute upper respiratory tract infections. Cochrane Database Syst Rev 2011:CD006895.

50 King S, Glanville J, Sanders ME, et al. Effectiveness of probiotics on the duration of illness in healthy children and adults who develop common acute respiratory infectious conditions: a systematic review and meta-analysis. Br J Nutr 2014;112:41-54.

51 Calder PC, Carr AC, Gombart AF. Optimal nutritional status for a Well-Functioning immune system is an important factor to protect against viral infections. Nutrients 2020;12.

52 Padhani ZA, Moazzam Z, Ashraf A. Vitamin C supplementation for prevention and treatment of pneumonia. Cochrane Database of Systematic Reviews.

53 Hemilä $\mathrm{H}$, Chalker $\mathrm{E}$. Vitamin $\mathrm{C}$ for preventing and treating the common cold. Cochrane Database Syst Rev 2013:CD000980 (Published Online First: 31 January 2013).

54 The Lancet respiratory medicine. COVID-19 testing in the UK. The Lancet Respiratory Medicine 2020;8:1061.

55 Griffith GJ, Morris TT, Tudball MJ, et al. Collider bias undermines our understanding of COVID-19 disease risk and severity. Nat Commun 2020;11:5749.

56 Surkova E, Nikolayevskyy V, Drobniewski F. False-Positive COVID-19 results: hidden problems and costs. Lancet Respir Med 2020;8:11671168.

57 HexaBiogen. DC-11-0004E | QuantiVirusTM real-time PCR coronavirus (SARS-CoV-2). QuantiVirusTM real-time PCR coronavirus (SARS-CoV-2) detection test, 2020. Available: https:// www.hexabiogen.com/sars-cov-2-produits-pour-le-diagnostic-5087/ quantivirus-real-time-pcr-coronavirus-701000077.html [Accessed 12 Oct 2020].

58 Mark HE, Dias da Costa G, Pagliari C, et al. Malnutrition: the silent pandemic. BMJ 2020;371:m4593.

59 NCT ClinicalTrials.gov. Identifier. 04579640, Trial of Vitamin D to Reduce Risk and Severity of COVID-19 and Other Acute Respiratory Infections (CORONAVIT. Bethesda (MD: National Library of Medicine (US), 2020. https://clinicaltrials.gov/ct2/show/NCT04579640 